\title{
Efficient Similarity Search within User-Specified Projective Subspaces
}

\author{
Michael E. Houle ${ }^{\mathrm{a}}$, Xiguo Ma ${ }^{\mathrm{b}}$, Vincent Oria ${ }^{\mathrm{c}}$, Jichao Sun ${ }^{\mathrm{c}}$ \\ ${ }^{a}$ National Institute of Informatics, Tokyo 101-8430, Japan \\ ${ }^{b}$ Google Mountain View, Mountain View CA 94043, USA \\ ${ }^{c}$ New Jersey Institute of Technology, Newark NJ 07102, USA
}

\begin{abstract}
Many applications - such as content-based image retrieval, subspace clustering, and feature selection - may benefit from efficient subspace similarity search. Given a query object, the goal of subspace similarity search is to retrieve the most similar objects from the database, where the similarity distance is defined over an arbitrary subset of dimensions (or features) - that is, an arbitrary axis-aligned projective subspace — specified along with the query. Though much effort has been spent on similarity search in fixed subspaces, relatively little attention has been given to the problem of similarity search when the dimensions are specified at query time. In this paper, we propose new methods for the subspace similarity search problem for real-valued data. Extensive experiments are provided showing very competitive performance relative to state-ofthe-art solutions.
\end{abstract}

Keywords: subspace similarity search, multi-step search, intrinsic dimensionality

\section{Introduction}

Similarity search is of great importance to applications in many different areas, such as data mining, multimedia databases, information retrieval, statistics and pattern recognition. Specifically, a similarity query retrieves from the database those objects that most closely resemble a specified query object, based on some measure of pairwise similarity (typically in the form of a distance function). Due to its importance, much effort has been spent on the efficient support of similarity search. However, most existing approaches consider search only with respect to a fixed feature space. In this paper, we focus on the subspace similarity search problem, in which the calculation of similarity values is restricted to a subset of dimensions specified along with the query object. By specifying a subset of dimensions, the user indicates that the similarity computation should be computed as if the data had been projected to a desired axis-parallel subspace beforehand. The challenge is to efficiently preprocess the data in such a way that any such projective similarity query can be accommodated effectively.

As with similarity search on fixed spaces, subspace similarity search may also have an impact in application areas where the feature set under consideration changes from operation to operation. Such changes could be due to a modification of query preferences (as in content-based

Email addresses: meh@nii.ac.jp (Michael E. Houle), maxiguo@google.com (Xiguo Ma), oria@njit. edu (Vincent Oria), js87@njit.edu (Jichao Sun)

Preprint submitted to Elsevier

December 1, 2015 
image retrieval), or to the determination of the local structure at different locations within data (as in subspace clustering), or to a systematic exploration of feature subspaces (as in feature selection). Motivated by the difficulty of search in higher dimensional spaces due to the so-called 'curse of dimensionality' $[1,2,3]$, the efficiency of similarity search may be improved through an appropriate projection to a lower-dimensional subspace (as in dimensional reduction). In contentbased image retrieval, images are often represented by feature vectors extracted based on color, shape, and texture descriptors. In an exploration of the data set, a query involving one combination of features (such as color) may be followed by a query on a different combination (such as shape). In subspace clustering [4], the formation of an individual cluster is generally assessed with respect to a subset of features that most closely describe the concept associated with the cluster. Since verification of a cluster requires the identification of a feature subset together with an object subset, the effectiveness of the overall clustering process may depend on the efficient processing of subspace similarity queries. Wrapper methods for feature selection [5] require an evaluation process, such as $k$-nearest neighbor $(k-\mathrm{NN})$ classification, for the identification of effective combinations of features. Exploration and visualization of feature subspaces [6, 7] can be extremely time-consuming when the neighborhoods are determined using exhaustive search, due to the exponential number of potential combinations involved. To accelerate the process, the efficient support of subspace similarity search is needed.

Almost all existing similarity search indices require that the similarity measure and associated vector space both be specified before any preprocessing occurs. Since the subspaces to be searched are typically not known until query time, traditional methods for fixed spaces (as surveyed in [8]) cannot be effectively applied for the subspace search problem. Even if the query subspaces were known in advance, constructing an index for every possible subspace would still be prohibitively expensive. Of all the methods for similarity search appearing in the research literature, only very few have been specifically formulated for the subspace search problem; a survey of these methods will be presented in Section 2.1. In general, existing solutions for subspace similarity search suffer greatly in terms of the computational cost.

Of the two main types of similarity queries ( $k$-NN queries and range queries), $k$-NN queries are often more important, due to the difficulty faced by the user in deciding range thresholds. This is especially the case for the search in subspaces, since the range values of interest will typically depend on the number of features associated with the subspace. In this paper, we focus only on $k$-NN queries.

We now formally define the subspace search problem for $k$-NN queries. Given an object domain $\mathcal{U}$, let $S \subseteq \mathcal{U}$ denote a set of database objects represented as feature vectors in $\mathbb{R}^{D}$. The set of features will be denoted simply as $F=\{1,2, \cdots, D\}$, with feature $i \in F$ corresponding to the $i$-th coordinate in the vector representation. Let $d: \mathbb{R}^{D} \times \mathbb{R}^{D} \rightarrow \mathbb{R}$ be a distance function defined for the vector space. Given an object vector $u=\left(u_{1}, \ldots, u_{|F|}\right) \in S$, its projection with respect to a feature subset $F^{\prime} \subseteq F$ is the vector $u^{\prime}=\left(u_{1}^{\prime}, \ldots, u_{|F|}^{\prime}\right)$ such that for all $i \in F$, $u_{i}^{\prime}=u_{i}$ whenever $i \in F^{\prime}$, and $u_{i}^{\prime}=0$ otherwise. The feature set $F^{\prime}$ thus indicates a unique axis-aligned projective subspace to which distance calculations can be restricted.

Definition 1 (Subspace $k$-NN Query). Given a query object $q \in \mathcal{U}$, a query subspace $F^{\prime} \subseteq F$, and a query neighborhood size $k$, a subspace $k-N N$ query $\left\langle q, F^{\prime}, k\right\rangle$ returns the $k$ objects of $S$ most similar to $q$, for the distance function $d_{F^{\prime}}(q, u) \triangleq d\left(q^{\prime}, u^{\prime}\right)$, where $q^{\prime}$ and $u^{\prime}$ are the projections of $q$ and $u$ with respect to $F^{\prime}$.

Note that with this definition of the problem, the user is given complete freedom to specify 
Table 1: Example of a subspace similarity query result for the choice of dimensions 2, 4, and 5, using the squared Euclidean distance.

\begin{tabular}{|c|c|c|c|c|c|c|c|c|c|c|c|c|}
\hline Dimension & $p_{1}$ & $p_{2}$ & $p_{3}$ & $p_{4}$ & $p_{5}$ & $p_{6}$ & $p_{7}$ & $p_{8}$ & $p_{9}$ & $p_{10}$ & Variance & $q$ \\
\hline 1 & 4.9 & 2.1 & 0.4 & 0.4 & 1.8 & 8.6 & 7.3 & 8.7 & 9.2 & 5.3 & 11.0481 & 9.0 \\
\hline 2 & 5.5 & 4.4 & 6.8 & 8.6 & 0.5 & 9.8 & 9.8 & 5.8 & 2.9 & 0.5 & 10.6324 & 5.5 \\
\hline 3 & 9.9 & 7.1 & 4.2 & 1.8 & 1.3 & 4.6 & 1.1 & 6.8 & 1.9 & 1.1 & 8.6016 & 1.0 \\
\hline 4 & 9.3 & 8.2 & 7.3 & 6.4 & 8.3 & 4.3 & 2.3 & 1.8 & 1.1 & 3.2 & 8.2856 & 8.7 \\
\hline 5 & 6.0 & 7.3 & 7.5 & 9.6 & 9.1 & 6.0 & 9.3 & 2.5 & 3.2 & 1.7 & 7.5496 & 6.5 \\
\hline Full dist \& & 96.63 & 86.92 & 88.85 & 99.11 & 83.85 & 51.22 & 58.39 & 97.43 & 76.26 & 91.99 & - & 0.0 \\
\hline$\frac{\text { rank to } q}{\text { dist \& }}$ & 8 & $\frac{5}{210}$ & $\begin{array}{c}6 \\
465\end{array}$ & $\frac{10}{2451}$ & 4 & $\frac{1}{3810}$ & $\frac{2}{55-49}$ & $\frac{9}{6370}$ & $\frac{3}{7541}$ & $\frac{7}{7829}$ & - & 0 \\
\hline $\begin{array}{l}\text { dist \& } \\
\text { rank to } q \\
\text { in } 2,4,5\end{array}$ & $\begin{array}{c}0.61 \\
1\end{array}$ & $\begin{array}{c}2.10 \\
2\end{array}$ & $\begin{array}{c}4.65 \\
3\end{array}$ & $\begin{array}{c}24.51 \\
4\end{array}$ & $\begin{array}{c}31.92 \\
5\end{array}$ & $\begin{array}{c}38.10 \\
6\end{array}$ & $\begin{array}{c}55.49 \\
7\end{array}$ & $\begin{array}{c}63.70 \\
8\end{array}$ & $\begin{array}{c}75.41 \\
9\end{array}$ & $\begin{array}{c}78.29 \\
10\end{array}$ & $\begin{array}{l}- \\
-\end{array}$ & $\begin{array}{c}0.0 \\
0\end{array}$ \\
\hline
\end{tabular}

any subspace with any number of dimensions. In particular, the number of possible choices of subspace in a $D$-dimensional full space is $2^{D}-1$.

As an example of a subspace distance function, for any given $p \in[1, \infty)$, the $L_{p}$ distance between two objects $q, u \in \mathcal{U}$ restricted to the axis-aligned projective subspace $F^{\prime}$ is defined as

$$
d_{F^{\prime}}(q, u)=\left(\sum_{i \in F^{\prime}}\left|q_{i}-u_{i}\right|^{p}\right)^{\frac{1}{p}} .
$$

Table 1 shows, for a set of 10 points in 5 dimensions, a 3-dimensional subspace similarity query result for the squared Euclidean distance.

The issues surrounding multidimensional query formulation, and in particular how users select data subspaces for exploration, are beyond the scope of this paper. Here, we limit our attention to the design of effective generic algorithms for the subspace similarity search problem. In particular, we follow the so-called 'multi-step' search strategy [9, 10,11], utilizing 1-dimensional distances as lower bounds to efficiently prune the search space. The main contributions of this paper are:

- algorithms specifically tailored for both exact and approximate axis-parallel subspace similarity search in real-valued data sets, where the subspace dimensions are specified along with the query;

- a guide to the practical choice of an important algorithm parameter, based on a theoretical analysis of sample properties;

- an experimental evaluation across data sets of a variety of types and sizes, showing the efficiency and competitiveness of our algorithms.

A preliminary version of these results was presented in [12].

The remainder of this paper is organized as follows. In Section 2 we give an overview of the related work on subspace search and multi-step search algorithms. Our proposed algorithms are presented in Section 3. In Section 4, through experiments on several real-world datasets, we contrast the performance of our methods with those of existing methods. The paper is concluded in Section 5.

\section{Related work}

In this section, we survey the research literature on subspace similarity search and multi-step search algorithms. 


\subsection{Subspace similarity search}

Relatively few similarity search methods exist that are specifically designed for subspace search. In [13], PVA (Partial VA-file) was proposed, which adapts the vector approximation file (VA-file) [3] to support subspace queries. The VA-file, designed for fixed-space similarity search, stores a compressed approximation of the data as a single file. At query time, the compressed approximation is scanned in its entirety, and the information thus obtained is used to derive upper and lower bounds of the true distances between data objects and the query object. These bounds are then used to either prune away candidates, or confirm their membership in the query result. For any candidate that cannot be pruned or confirmed using the upper and lower distance bounds, its membership is decided by accessing its full vector representation, and using this vector to compute its true distance to the query. PVA, on the other hand, stores an approximation of data on each dimension separately, and processes the search using only those 1-dimensional VA-files that correspond to dimensions involved in the query.

In [14], DMI (Dimension-Merge Index) was developed, which combines multiple 1-dimensional index structures to answer subspace queries. DMI builds an index for each dimension separately (of any desired type), and utilizes these indexes with respect to the query dimensions to perform the search. Specifically, in a round-robin fashion, DMI retrieves candidates for the query result from the neighborhood associated with each of the query dimensions, while maintaining a lower bound for the smallest of the subspace distances to objects that have not yet been visited. The algorithm terminates when at least $k$ of the visited objects have subspace distances no greater than the lower bound. Since no unseen objects would have subspace distances smaller than the lower bound, the algorithm is guaranteed to return an exact query result.

In [15], PT (Projected R-tree) was proposed as a redefinition of the classical search structure R-tree [16] for subspace similarity search. Instead of integrating results of queries on 1dimensional indices, PT utilizes a single index designed for the full feature space (an R-tree) to answer queries with respect to subspaces. In PT, as with R-tree indexing, groups of nearby objects are represented by their minimum bounding rectangle at the next higher level of the tree. Since between the query object $q$ and minimum bounding rectangles, the minimum distance in subspace $F^{\prime}$ is properly defined, the R-tree best-first search approach can be applied without changes. The minimum distance ( $L_{p}$ distance) between a minimum bounding rectangle $\mathcal{R}$ and the query object $q$ in subspace $F^{\prime}$ is defined as

$$
\operatorname{mindist}_{F^{\prime}}(q, \mathcal{R})=\left(\sum_{i \in F^{\prime}}\left\{\begin{array}{ll}
\left(\mathcal{R}_{i}^{\min }-q_{i}\right)^{p} & \text { if } \mathcal{R}_{i}^{\min }>q_{i} \\
\left(q_{i}-\mathcal{R}_{i}^{\max }\right)^{p} & \text { if } \mathcal{R}_{i}^{\max }<q_{i} \\
0 & \text { otherwise }
\end{array}\right)^{\frac{1}{p}},\right.
$$

where $\mathcal{R}_{i}^{\min }$ and $\mathcal{R}_{i}^{\max }$ are the minimum and maximum coordinate values of rectangle $\mathcal{R}$ in the $i$-th dimension.

PVA, DMI and PT all produce exact query results; however, as we shall see in Section 4, all tend to suffer greatly in terms of their computational costs.

Another approach to the subspace search problem was proposed by Lian and Chen in [17], for range queries. Here, the search space is reduced through the application of the triangle inequality on several pivot points. Since $k$-NN queries are not directly supported by this algorithm, for the experimental comparison in Section 4, we restrict our attention to PVA, DMI and PT.

\subsection{Multi-step search algorithms}

Our proposed solutions for the subspace search problem make use of multi-step search algorithms. Multi-step search was originally proposed for the adaptive similarity search problem, 
which aims to find the most similar objects to a query object $q$ from the database $S$ with respect to an adaptive similarity measure - that is, a similarity measure that can be determined by the user at query time. Multi-step search computes a query result using a fixed 'lower-bounding' distance function that is adapted to answer the same query with respect to a user-specified 'target' distance function. The function $d_{l}$ is a lower-bounding distance for the target distance $d_{t}$ if $d_{l}(u, v) \leq d_{t}(u, v)$ for any two objects $u, v$ drawn from a domain for which both $d_{l}$ and $d_{t}$ are defined. In multi-step search, there are two main stages: filtering and refinement. In the filtering stage, from the database $S$, a candidate set is generated using $d_{l}$. In the refinement stage, the candidate set is refined using $d_{t}$ to obtain the query result.

The first multi-step $k$-NN search algorithm was proposed by Korn et al. [9]. In the filtering stage, the algorithm first obtains from the database $S$ the $k$-nearest neighbors of $q$ with respect to $d_{l}$, and then computes their distances to $q$ with respect to the target distance $d_{t}$. From among the target distance values, the maximum value $d_{\max }$ is determined. The candidate set is then obtained from $S$ by performing a range query with respect to $d_{l}$, where $d_{\max }$ is used as the range limit. In the refinement stage, the algorithm simply evaluates the target distances from $q$ to all the candidates to get the query result. The algorithm guarantees a correct query result; however, the candidate set produced may be prohibitively large.

Later, Seidl and Kriegel [10] proposed a more efficient multi-step algorithm, which we will refer to as SK in the remainder of this paper. The description of algorithm SK is presented in Figure 1. In contrast to the first multi-step $k$-NN search algorithm, SK performs rounds of filtering and refinement on a candidate set that grows incrementally. The function getnext allows for incremental generation of the neighbors of $q$ with respect to $d_{l}$. For most search indexes, this can be done with simple bookkeeping of the search state. With the bookkeeping, the search for the $(j+1)$-th nearest neighbor can be resumed from the saved state after the termination of the search for the $j$-th nearest neighbor.

At every iteration of the main loop of algorithm SK, the candidate set is the current neighborhood of $q$ with respect to $d_{l}$. From the candidate set, the $k$-nearest neighbors of $q$ with respect to $d_{t}$ are stored as a tentative query result (the set of object-distance pairs $P$ ), and the $k$-th smallest target distance is maintained $\left(d_{\max }\right)$. The algorithm terminates when the value of $d_{\max }$ is no greater than the largest lower-bounding distance that has been seen so far $\left(\beta_{l}\right)$, or when all the objects in the data set $S$ have been fetched as candidates and refined.

In [10], Seidl and Kriegel showed that algorithm SK is optimal in that it produces the minimum number of candidates needed in order to guarantee a correct query result, given only a list of candidates ordered according to $d_{l}$. In other words, with even one such candidate missing, the query result may not be guaranteed correct.

\subsection{MAET+}

Despite its performance guarantee, the SK algorithm may still be expensive in practice. Using SK as a starting point, Houle et al. [11] designed an approximate multi-step algorithm, MAET, which utilizes tests of a measure of the local intrinsic dimensionality of the data, the generalized expansion dimension (GED) $[18,19]$, to guide early termination decisions.

In contrast with previous multi-step algorithms, MAET also generalizes the lower-bounding relationship between $d^{\prime}$ and $d$, by allowing a relaxation of the lower bound: given some lowerbounding ratio $\lambda \geq 1$, the lower-bounding distance need only satisfy $\lambda \cdot d^{\prime}(u, v) \leq d(u, v)$ for any two objects $u, v \in \mathcal{U}$. The motivation for introducing $\lambda$ is to allow as tight as possible a fit between the lower-bounding distance and the target distance. In practice, it might be difficult for 
Table 2: Notation used in this paper.

\begin{tabular}{|l|l|}
\hline Symbol & Description \\
\hline$S$ & database $S \subseteq \mathcal{U}$ \\
$k$ & target query result size \\
$d_{t}(u, v)$ & distance between two objects $u$ and $v$ in target subspace \\
$d_{l}(u, v)$ & lower-bounding distance between two objects $u$ and $v$ \\
$N_{F^{\prime}}(q, k)$ & set of $k$-nearest neighbors of $q$ with respect to feature subset $F^{\prime}$ \\
$\delta_{F^{\prime}}(q, k)$ & the $k$-th smallest subspace distance from $q$ with respect to $F^{\prime}$ \\
$\lambda$ & lower-bounding ratio \\
$\lambda_{e}$ & estimate of lower-bounding ratio \\
$P$ & set of candidate pairs $(v, \beta)$, where $v$ is an object and $\beta$ is a distance \\
$P_{v}$ & candidate object set associated with $P$ \\
\hline
\end{tabular}

the user to determine a value of the lower-bounding ratio $\lambda$ with which MAET can perform well. If the ratio is too small, a larger candidate set can be expected; if it is too large, the accuracy of the query result might not be satisfied.

In [11], a variant MAET+ is also proposed in which an appropriate value of $\lambda$ is dynamically estimated from observed distance values. After each candidate $v$ and lower-bounding distance $\beta^{\prime}=d^{\prime}(q, v)$ is retrieved from the underlying index, the ratio of the target distance $\beta=d(q, v)$ to $\beta^{\prime}$ is computed, and the smallest ratio encountered thus far is stored in $\lambda_{e}$ as the estimate of $\lambda$. A description of MAET+ is presented in Figure 2. Compared to the SK algorithm [10], the MAET and MAET+ algorithms are able to obtain significant improvements in terms of the computational cost, while losing very little in the accuracy of the query result.

The local intrinsic dimensionality score GED can be regarded as a measure of the expansion of data in the vicinity of a query point $q$, defined in terms of the numbers of data points captured by two nested balls $B\left(q, r_{1}\right)$ and $B\left(q, r_{2}\right)$ centered at $q$, with differing radii $r_{1}$ and $r_{2}$, respectively:

$$
\operatorname{GED}\left(B\left(q, r_{1}\right), B\left(q, r_{2}\right)\right)=\frac{\log \left|B\left(q, r_{1}\right)\right|-\log \left|B\left(q, r_{2}\right)\right|}{\log r_{2}-\log r_{1}},
$$

where $|B(q, r)|$ denotes the number of data points captured by the ball $B(q, r)$. In [11], an analysis of the performance of MAET and MAET+ is given in terms of the maximum $\operatorname{Max} \operatorname{GED}(q, k)$ of the values of $\operatorname{GED}\left(B_{i}, B_{k}\right)$ over all choices of $1 \leq i<k$, where for $1 \leq j \leq k, B_{j}$ is the ball centered at $q$ with radius equal to the $j$-nearest-neighbor distance from $q$.

Theorem 1 ([11]). Let $\mathcal{U}$ be a data domain upon which distance functions $d$ and $d^{\prime}$ are defined, with $\lambda d^{\prime}(u, v) \leq d(u, v)$ for all $u, v \in \mathcal{U}$ where $\lambda \geq 1$. Let $q \in \mathcal{U}, k>0, \lambda$ and $t>0$ be the inputs provided to a call to Algorithm MAET or MAET+. If $\operatorname{Max} \operatorname{GED}(q, k+1)$ is defined, then the algorithm returns the correct query result whenever $t \geq \operatorname{Max} G E D(q, k+1)$. Otherwise, the algorithm returns the correct query result regardless of the value of $t$.

\section{Algorithm}

We now present our solutions to the subspace similarity search problem. Let us first introduce some additional notation (for a summary, see Table 2). For any object $q \in \mathcal{U}$ and any subspace 


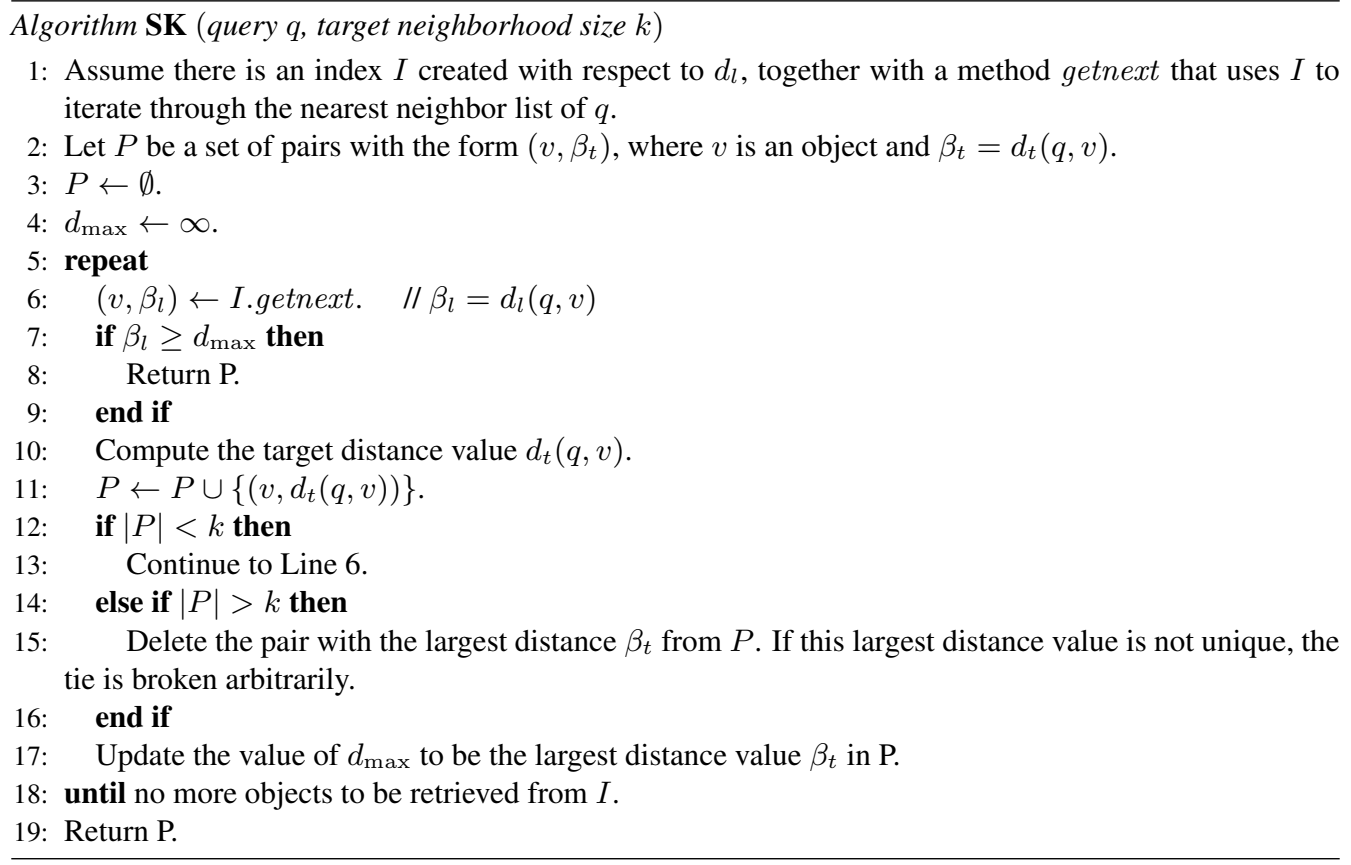

Figure 1: The description of the exact multi-step similarity search algorithm SK (from [11]). The algorithm examines candidates in increasing order of lower-bounding distances, while maintaining a top- $k$ result set in terms of the target distance. If the lower-bounding distance meets or exceeds the target distances to all members of the current result set, the search terminates. 


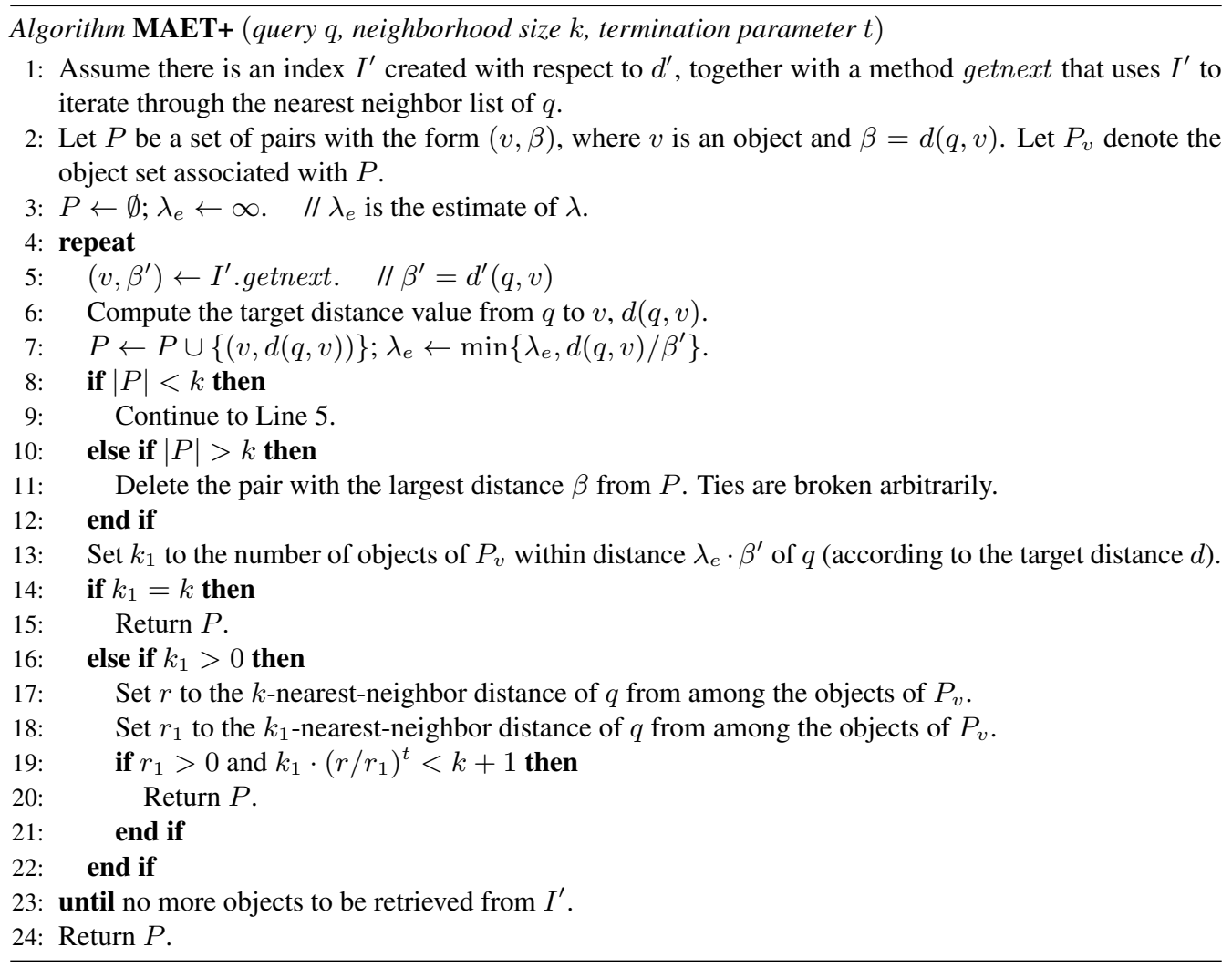

Figure 2: The description of the approximate multi-step similarity search algorithm MAET+ (adapted from [11]), which dynamically determines the lower-bounding ratio $\lambda$ from observed distance values.

$F^{\prime} \subseteq F$, let $N_{F^{\prime}}(q, k)$ denote the $k$-nearest neighbor $(k$-NN) set of $q$ within database $S$ with respect to subspace distance $d_{F^{\prime}}$. Note that if the neighbor distances are not unique, the $k$-NN set may not be unique. In this case, in order to specify a unique $k$-NN set, we assume that ties in distance values are broken arbitrarily but consistently. Let $\delta_{F^{\prime}}(q, k)$ denote the $k$-th smallest subspace distance (with respect to $F^{\prime}$ ) from $q$ to the objects in $S$.

The strategy underlying our methods involves the application of multi-step search, using a lower-bounding distance function to filter a candidate set from the database, and using the target distance function to refine the candidate set to obtain the final query result. The main concern here is the determination at query time of a lower-bounding distance function suitable for the indicated subspace. Due to the exponential number of possible subspaces, it is impossible to explicitly preprocess the data for every subspace. Instead, as potential lower-bounding distance functions, we consider only the 1-dimensional distance $d_{\{i\}}$ associated with each feature $i \in F$. Assuming that the lower-bounding property holds between $d_{\{i\}}$ and subspace distance $d_{F^{\prime}}$ for all $i \in F^{\prime}$ (which is the case for many practical distance measures, including the Euclidean and squared Euclidean distances, regardless of the number of the total number of features $|F|$ ), there are $\left|F^{\prime}\right|$ lower-bounding distance functions that can be used in the search. However, practical performance may vary considerably according to the choice of $d_{\{i\}}$. In order to minimize the 
Algorithm SK_SR (query q, subspace $F^{\prime}$, target neighborhood size $k$ )

// Preprocessing step: obtain a single ranking of all dimensions.

1: for each dimension $i \in F$ do

2: $\quad \mu_{i} \leftarrow \frac{1}{|S|} \sum_{u \in S} u_{i}$.

3: $\quad \operatorname{Var}_{i} \leftarrow \frac{1}{|S|} \sum_{u \in S}\left(u_{i}-\mu_{i}\right)^{2}$.

4: end for

5: Rank all dimensions $i \in F$ in decreasing order of $\operatorname{Var}_{i}$. Let $\Re(F)$ denote this ranking. // Query processing step: perform a multi-step search.

6: Among all the dimensions in subspace $F^{\prime}$, select the dimension $i^{*}$ with the highest ranking according to $\Re(F)$.

7: Call $\operatorname{SK}(q, k)$ to produce the query result, with $d_{\left\{i^{*}\right\}}$ as the lower-bounding distance function, and $d_{F^{\prime}}$ as the target distance function.

Figure 3: The description of subspace similarity search algorithm SK_SR, which uses a single overall ranking of variance to determine the lower-bounding dimension.

risk of choosing a poorly-performing lower-bounding distance, we select the distance function corresponding to the most discriminative query dimension. This is done by ranking the dimensions based on data variance, a simple yet effective ranking technique. Two ranking strategies are proposed in this paper: Single Ranking (SR) and Multiple Ranking (MR).

\subsection{Single ranking strategy}

The first of our proposed algorithms - SK_SR, described in Figure 3 - employs a single overall ranking of dimensions based on variance. There are two main phases: a preprocessing phase and a query processing phase. In the preprocessing phase, the algorithm generates a single ranking of the dimensions, in terms of the variances of the data values computed separately for each of the dimensional coordinates - the larger the data variance for a given dimension, the higher the ranking of that dimension. In the query processing phase, as the lower-bounding distance function used in multi-step search, the algorithm chooses the dimension of highest rank from among the query dimensions. For the example shown in Table 1 , in which a similarity query is performed over the set of dimensions $\{2,4,5\}$, lower-bounding distances would be computed along dimension 2 , since the feature values for this dimension have higher variance than those of dimensions 4 and 5.

When Algorithm SK is used for performing the multi-step search (in Line 7), the query result is guaranteed to be correct. As an alternative, we may also utilize the approximate multi-step algorithm MAET+; this variant of subspace similarity search will be referred to as MAET+_SR. Specifically, we make a call to MAET $+(q, k, t)$, where $t>0$ is a parameter governing an early termination criterion. Larger choices of $t$ can be expected to yield query results with higher accuracies at the possible expense of computational cost. In [11], a sampling method was designed for choosing $t$ so that a desired proportion of potential queries can be correctly answered with high probability.

Note that like DMI, our search strategy requires the construction of a separate index for each of the dimensions. However, unlike DMI, our algorithms access only a single index per query, namely the most discriminative query dimension in terms of variance. 


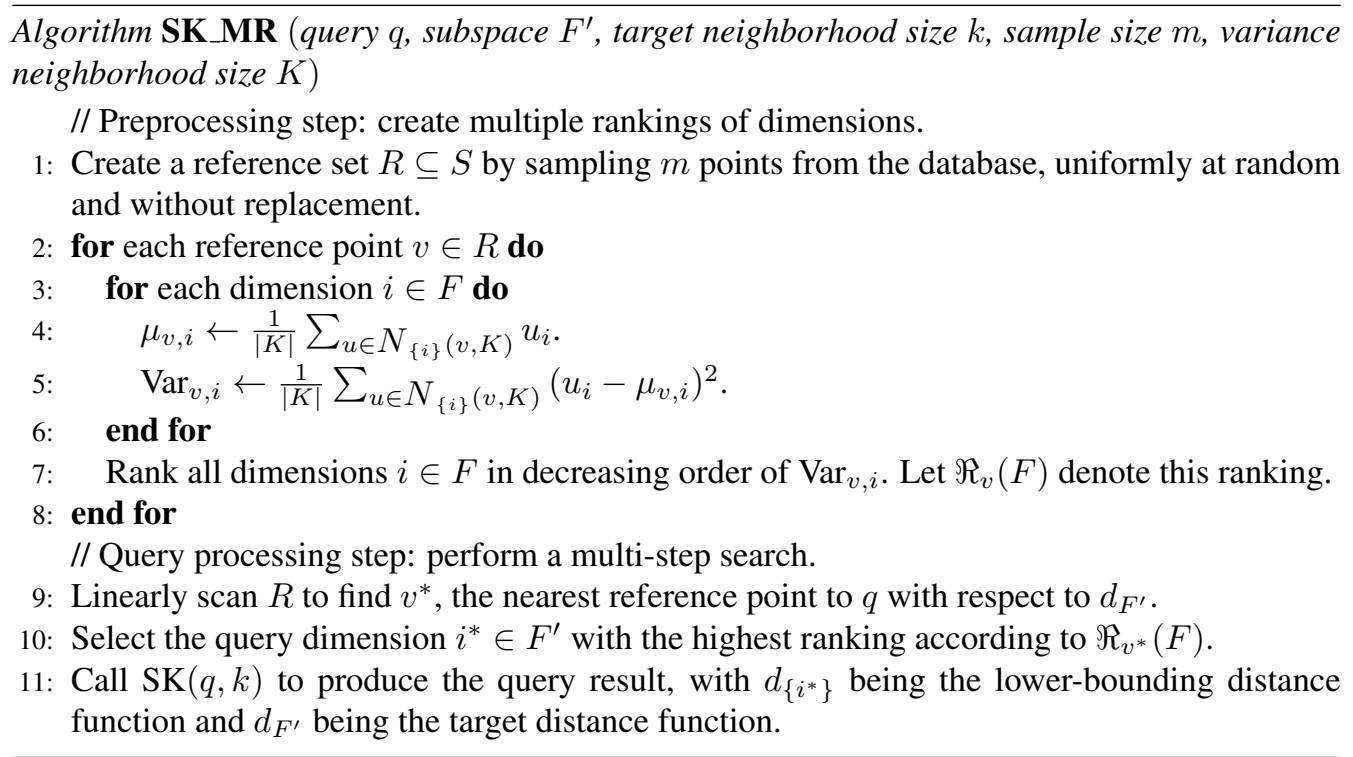

Figure 4: The description of subspace similarity search algorithm SK_MR, which uses rankings of variance with respect to multiple reference objects to determine the lower-bounding dimension.

\subsection{Multiple ranking strategy}

The single ranking strategy has the advantage of being straightforward to apply. However, its effectiveness may be limited whenever the variance of a particular dimension differs greatly when restricted to the vicinity of differing query objects. For this reason, we have also designed a multiple ranking strategy that takes the query object into account when generating a ranking of dimensions.

Our multiple ranking strategy for subspace similarity search, SK_MR, is described in Figure 4. In the preprocessing step, the algorithm first samples $m$ reference points from the database. Then, with respect to each reference point $v$, the algorithm determines a ranking (from highest to lowest) of all dimensions based on the variance of the coordinate values for the dimension in question, this time computed over a neighbor set of $v$ (instead of over the entire dataset $S$ ). In the query processing step, the algorithm first finds the nearest reference point $v^{*}$ of $q$ in the query subspace (using sequential search within the reference set), and then uses the ranking of dimensions precomputed for $v^{*}$ in the processing of query $q$. Again, we may replace SK with $\mathrm{MAET}+$ to derive an approximation variant, MAET+_MR.

Two parameter choices must be considered when applying the multiple ranking strategy: the number of reference points $m$, and the size $K$ of the neighborhoods within which data variance is computed. As will be shown in Section 4, the choice of $K$ does not greatly affect the performance, provided that it is small relative to the dataset size $|S|$. On the other hand, the number of reference points $m$ must be chosen with more care. If $m$ is too large, the identification of the most discriminative query dimension may become unaffordable. If $m$ is too small, the dimension $i^{*}$ selected for multi-step search may not be very discriminative for the query. We next discuss how to choose a reasonable value for $m$.

Determining the Reference Set Size. For the multiple ranking strategy to be effective, for 
any given query point $q$, its nearest reference point $v^{*}$ should be among the nearest neighbors of $q$ within $S$ (all with respect to the query subspace). Otherwise, the ranking of dimensions based at $v^{*}$ may fail to approximate the ranking based at $q$. Fortunately, the following technical lemma shows that with even a relatively small number of reference points, $v^{*}$ can lie in the local neighborhood of $q$ with high probability.

Lemma 1 (Houle et al. [11]). Let $A$ be a set of positive integers, and let $A^{\prime} \subseteq A$ be a subset sampled uniformly at random without replacement. Given a threshold $\tau$, let a and $a^{\prime}$ refer to the number of elements in $A$ and $A^{\prime}$, respectively, that are no greater than $\tau$. Take $\eta$ and $\eta^{\prime}$ to refer to the proportion of these elements within $A$ and $A^{\prime}$, respectively. For any real number $\phi \geq 0$, we have the probability bound $\operatorname{Pr}\left[\left|\eta-\eta^{\prime}\right| \geq \phi\right] \leq 2 e^{-2 \phi^{2}\left|A^{\prime}\right|}$.

Proof 1. Since $A^{\prime}$ is generated by uniform selection from $A$, random variable $a^{\prime}$ follows the hypergeometric distribution with expectation $\mathrm{E}\left[a^{\prime}\right]=a\left|A^{\prime}\right| /|A|$. In [20], Chvátal showed that random variable $a^{\prime}$ satisfies both $\operatorname{Pr}\left[\mathrm{E}\left[a^{\prime}\right] \geq a^{\prime}+\phi\left|A^{\prime}\right|\right] \leq e^{-2 \phi^{2}\left|A^{\prime}\right|}$ and $\operatorname{Pr}\left[\mathrm{E}\left[a^{\prime}\right] \leq a^{\prime}-\phi\left|A^{\prime}\right|\right] \leq$ $e^{-2 \phi^{2}\left|A^{\prime}\right|}$. Both inequalities can be combined to yield the following error bound:

$$
\begin{aligned}
\operatorname{Pr}\left[\left|\eta-\eta^{\prime}\right| \geq \phi\right] & =\operatorname{Pr}\left[\left|\frac{a}{|A|}-\frac{a^{\prime}}{\left|A^{\prime}\right|}\right| \geq \phi\right]=\operatorname{Pr}\left[\left|\frac{\mathrm{E}\left[a^{\prime}\right]}{\left|A^{\prime}\right|}-\frac{a^{\prime}}{\left|A^{\prime}\right|}\right| \geq \phi\right] \\
& =\operatorname{Pr}\left[\left|\mathrm{E}\left[a^{\prime}\right]-a^{\prime}\right| \geq \phi\left|A^{\prime}\right|\right] \leq 2 e^{-2 \phi^{2}\left|A^{\prime}\right|} .
\end{aligned}
$$

To apply this lemma to the analysis of the choice of reference set size, let $A=\{1,2, \ldots,|S|\}$ represent the ranks of all the objects in $S$ with respect to a query object $q$, and let $A^{\prime} \subseteq A$ store the ranks of all the reference points $\left(\left|A^{\prime}\right|=m\right)$. Also, let $\tau$ be the rank of the reference point $v^{*}$, which implies that $\eta^{\prime}=1 /\left|A^{\prime}\right|$. A small value of $\eta$ would therefore indicate that $v^{*}$ is in the local neighborhood of $q$, as desired. From Lemma 1, we know that the probability of $\eta$ deviating from $\eta^{\prime}=1 /\left|A^{\prime}\right|$ by more than $\phi \geq 0$ is at most $2 e^{-2 \phi^{2}\left|A^{\prime}\right|}$. That is, the probability of $\eta$ being significantly larger than $1 /\left|A^{\prime}\right|$ vanishes quickly as the sample size $\left|A^{\prime}\right|$ grows. In practice, even small sample sizes allow us to obtain reasonably small values of $\eta$ with high probability. For example, if $\left|A^{\prime}\right|=5,000$ and $\phi=0.02$, the lemma indicates that the probability of $\eta \geq 0.0202$ is at most 0.037 , or equivalently, the probability of $\eta<0.0202$ is at least 0.963 .

\section{Experimental results}

In this section, we present the results of our experimentation. We compared our algorithms with the state-of-the-art approaches PVA, PT and DMI.

\subsection{Experimental framework}

\subsubsection{Data sets.}

Five publicly-available data sets were considered for the experimentation, so as to compare across a variety of set sizes, dimensions and data types.

- The Amsterdam Library of Object Images (ALOI) [21] consists of 110, 250 images of 1000 small objects taken from different viewpoints and illumination directions. The images are represented by 641-dimensional feature vectors based on color and texture histograms (for a detailed description of the image features, see [22]). 
- The MNIST data set [23] consists of 70, 000 images of handwritten digits from 500 different writers, with each image represented by 784 gray-scale texture values.

- The Cortina data set [24] consists of 1,088,864 images gathered from the World Wide Web. Each image is represented by a 74-dimensional feature vector based on homogeneous texture, dominant color and edge histograms.

- The Forest Cover Type set (FCT) [25] consists of 581,012 data points, with each representing a $30 \times 30$ square meter area of forest. Each point is represented by 54 attributes, associated with elevation, aspect, slope and other geographical characteristics.

- The ANN_SIFT data set [26] consists of $10^{7}$ SIFT descriptors [27] of 128 dimensions. The SIFT descriptors were extracted from approximately $10^{6}$ general images.

\subsubsection{Methodology.}

For each test, 1000 queries were generated at random, each consisting of an object $q$ selected from the database, and a query subspace $F^{\prime}$. Unless stated otherwise, the number of query dimensions was $\left|F^{\prime}\right|=8$, and the target neighborhood size was $k=10$. The Euclidean distance was used for all experiments. Two quantities were measured for the evaluation: query result accuracy and execution time. The results were reported as averages over the 1000 queries performed. The execution time is shown as a proportion of the time needed for a sequential search of the entire dataset. For each query, the accuracy of its $k$-NN result is defined as the proportion of the result falling within the true $k$-NN (subspace) distance to $q$ :

$$
\frac{\left|\left\{v \in Y \mid d_{F^{\prime}}(q, v) \leq \delta_{F^{\prime}}(q, k)\right\}\right|}{k},
$$

where $Y$ denotes the $k$-NN query result of $q$ in subspace $F^{\prime}(|Y|=k)$. For example, if the true 5 -NN distance to $q$ were 1.0 , then an approximate 5-NN query result achieving the distances $\{0.1,0.7,1.0,1.0,1.2\}$ would have an accuracy of 0.8 .

\subsection{Effects of varying $m$ and $K$ on the multiple ranking strategy}

For the first set of experiments, for all of the datasets under consideration, we tested the effects on the multiple ranking strategy due to variation of the sample size $m$ and variance neighborhood size $K$. When varying the sample size $m$, the variance neighborhood size $K$ was chosen to be approximately $1 \%$ of the dataset size: specifically, the choices were $K=10^{3}$ for ALOI and MNIST, $K=10^{4}$ for Cortina and FCT, and $K=10^{5}$ for ANN_SIFT. When varying $K$, the sample size $m$ was fixed at 500 for all datasets tested.

The results for varying $m$ on the datasets ALOI, MNIST, Cortina, FCT, and ANN_SIFT are shown in Figure 5. Here, we see that $m=500$ is a sufficiently-large sample size for the multiple ranking strategy to be effective, which is better than indicated by the theoretical analysis. From Lemma 1 , we know that if $m=500$, then for any dataset with any number of data points, the probability of $\eta<0.062$ is at least $0.945(\phi=0.06)$. Recall that the effectiveness of the multiple ranking strategy is expected to increase as $\eta$ diminishes. Our experimental findings show that the value of $\eta$ in practice is typically much smaller than what the analysis indicates. In order to reduce the computational cost of the experimentation, we therefore set $m=500$ for all remaining experiments.

The results for varying $K$ on the datasets ALOI, MNIST, Cortina, FCT, and ANN_SIFT are shown in Figure 6. As expected, the variance neighborhood size $K$ does not greatly affect the 


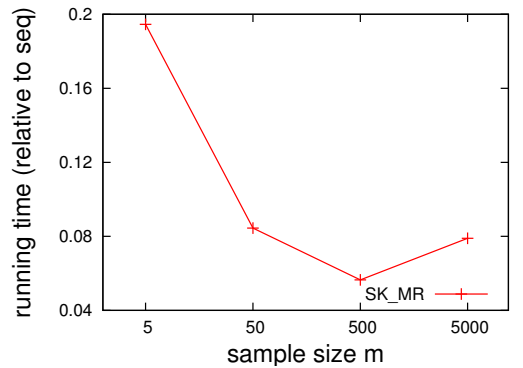

(a) ALOI, $K=10^{3}$

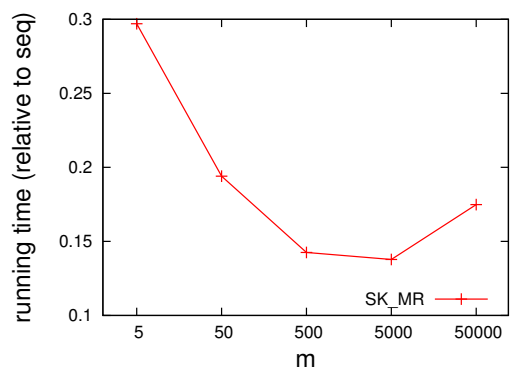

(c) Cortina, $K=10^{4}$

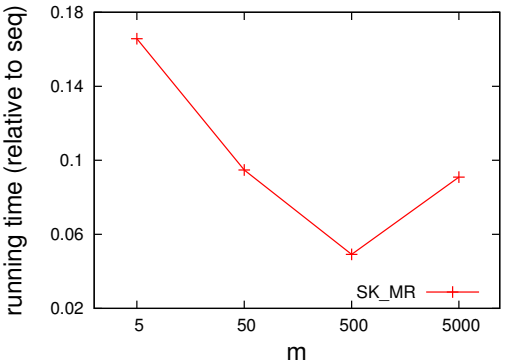

(b) MNIST, $K=10^{3}$

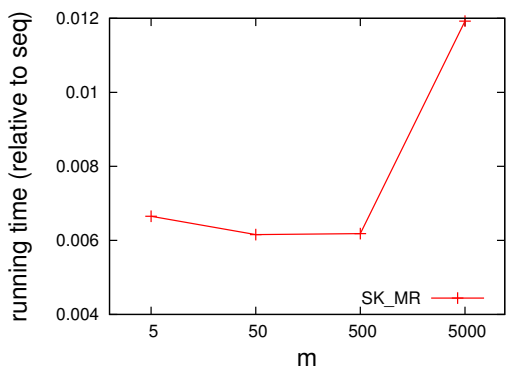

(d) FCT, $K=10^{4}$

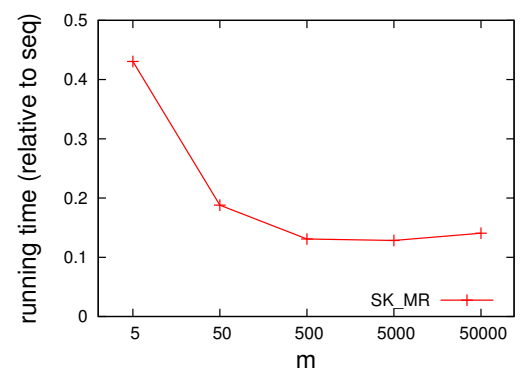

(e) ANN_SIFT, $K=10^{5}$

Figure 5: Experimental results obtained for the multiple ranking strategy when varying the sample size $m$. The results show that $m=500$ is a sufficiently large sample size for multiple ranking to be effective. The execution times are shown as a proportion of the time required for an execution of sequential search. 


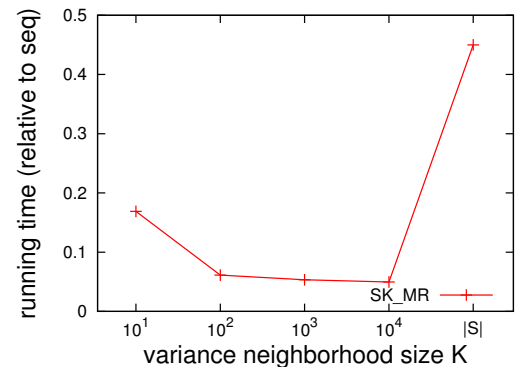

(a) ALOI, $m=500$

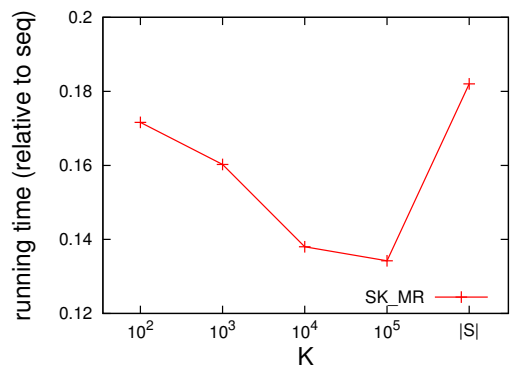

(c) Cortina, $m=500$

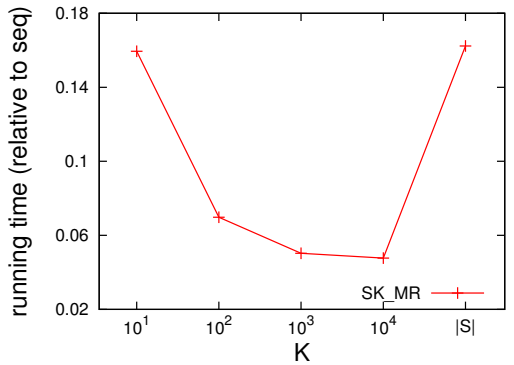

(b) MNIST, $m=500$

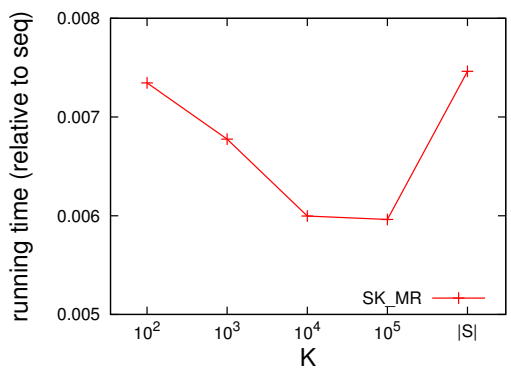

(d) FCT, $m=500$

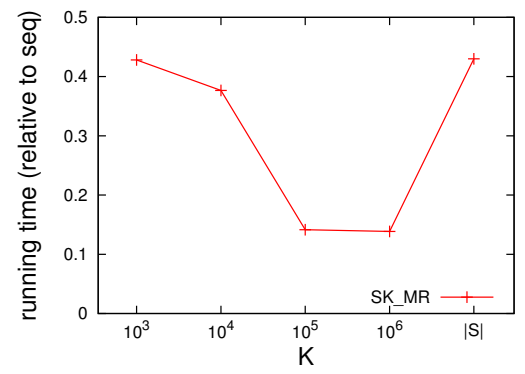

(e) ANN_SIFT, $m=500$

Figure 6: Experimental results obtained for the multiple ranking strategy over a range of values of the variance neighborhood size $K$. The results show that performance is generally stable over a very wide range of $K$, provided that it is reasonably small relative to the data set size. 


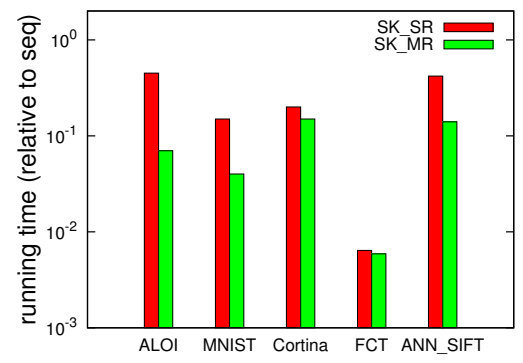

Figure 7: The comparison of Single Ranking (SR) and Multiple Ranking (MR) on all datasets tested.

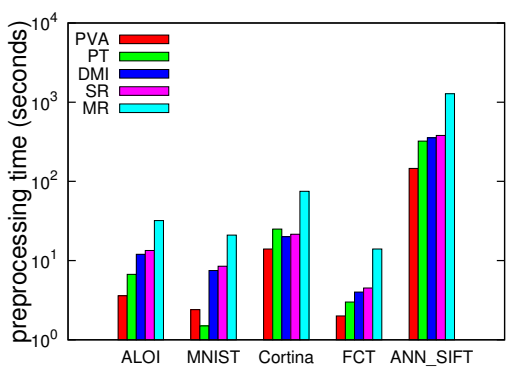

Figure 8: Preprocessing costs for all datasets tested.

performance, provided that it is set to reasonably small values relative to the dataset size. For all remaining experiments, we set $K=10^{3}$ for ALOI and MNIST, $K=10^{4}$ for Cortina and FCT, and $K=10^{5}$ for ANN_SIFT.

\subsection{Comparison of single ranking and multiple ranking}

We next compared the performance of the Single Ranking and Multiple Ranking strategies; the results are shown in Figure 7. Unsurprisingly, MR outperformed SR for all datasets tested. For the ALOI, MNIST, and ANN_SIFT sets, the query execution time of MR improved over that of SR by at least a factor of 3 - for all three sets, MR allows a speedup over sequential search of roughly one order of magnitude. For the Cortina set, the improvement was less dramatic, yet still substantial. Both methods performed excellently for the FCT set, achieving a more than two order of magnitude speedup over sequential search. Nevertheless, for this set as well, MR was seen to outperform SR.

As SR has been shown to have no significant advantages over MR, in order to simplify the comparison of our methods with its competitors, we shall omit the experimental results for SR from the performance plots presented in the remainder of the paper.

\subsection{Comparison with other methods}

We conducted two sets of experiments for the comparison of our algorithms with competing methods, varying each of two parameters in turn: the number of subspace dimensions $\left|F^{\prime}\right|$, and the target neighborhood size $k$. Specifically, we varied $\left|F^{\prime}\right|$ from 2 to 32 while fixing $k=10$, and varied $k$ from 5 to 40 while fixing $\left|F^{\prime}\right|=8$.

The results of varying $\left|F^{\prime}\right|$ are shown in Figure 9. For all datasets and all choices of $\left|F^{\prime}\right|$, our proposed methods generally outperform their competitors. Among all the methods tested, PVA is the most expensive, perhaps due to its use of sequential scan.

PT utilizes an R-tree built on the full-dimensional space to answer queries in subspaces; consequently, one would expect it to be less effective for subspaces in which $d_{F^{\prime}}$ differs greatly from $d_{F}$. This can explain the improvement in the performance of PT as the number of subspace dimensions increases. Nevertheless, due to the limits on the performance of R-trees for spaces of even moderate dimensionality, PT will still become prohibitively expensive as the number of subspace dimensions grows.

DMI processes queries by aggregating partial results across neighborhoods with respect to every query dimension. The aggregation may become prohibitively expensive as the number 


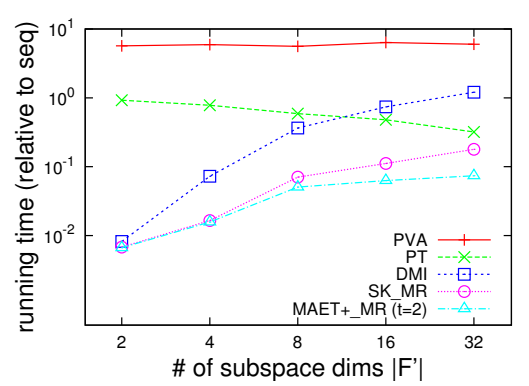

(a) ALOI

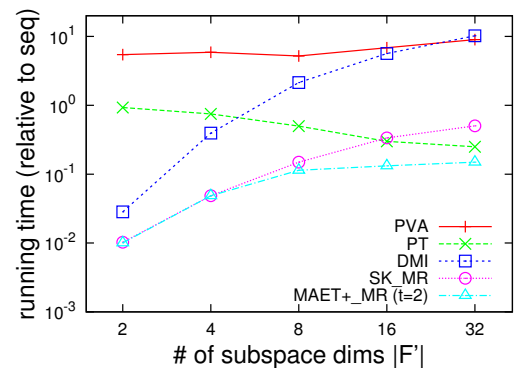

(c) Cortina

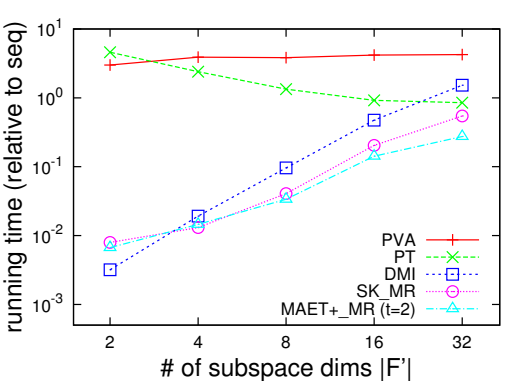

(b) MNIST

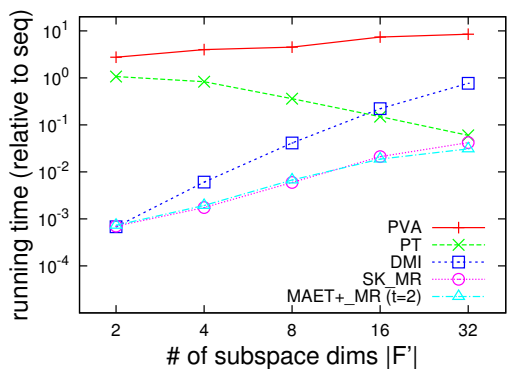

(d) FCT

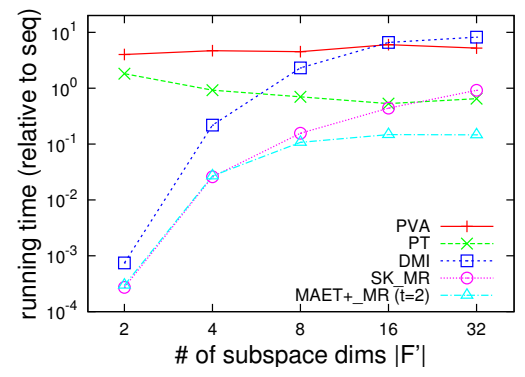

(e) ANN_SIFT

Figure 9: The results of varying $\left|F^{\prime}\right|$ on all tested datasets, with $k=10$. The results are exact, except for those of MAET+_MR. The average accuracies of MAET+_MR with $t=2$ are approximately $92 \%, 90 \%, 88 \%, 97 \%$ and $90 \%$ for ALOI, MNIST, Cortina, FCT and ANN_SIFT, respectively.

of subspace dimensions increases. In contrast, our algorithms avoid expensive aggregation by restricting the processing to a single query dimension.

Relative to SK_MR, we observe that for high subspace dimensionality, MAET+_MR can achieve a significant improvement in running time while still achieving a high level of accuracy. We note that as the value of $\left|F^{\prime}\right|$ increases, the computational cost of all tested methods must eventually tend to that of sequential search, as one would expect due to the curse of dimensionality.

Figure 10 shows the results of varying $k$. Again, our proposed methods generally outperform their competitors, with MAET+_MR achieving a slight improvement in running time over SK_MR, at the cost of a slight loss of accuracy. We also observe that the behaviors of all tested 


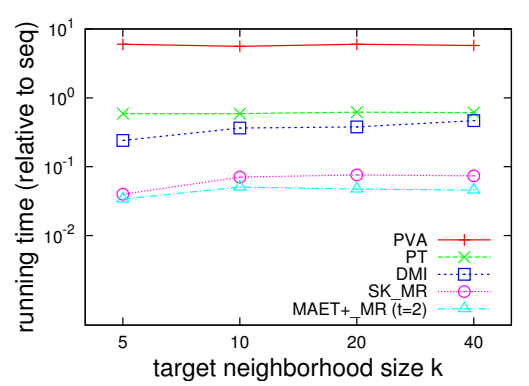

(a) ALOI

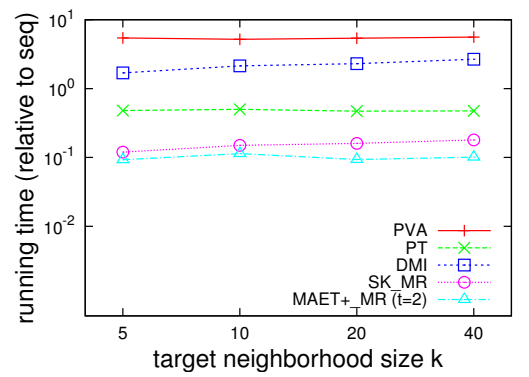

(c) Cortina

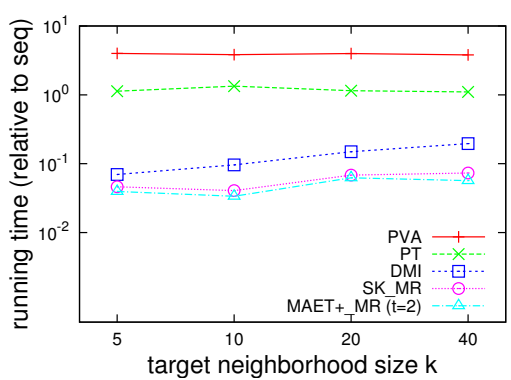

(b) MNIST

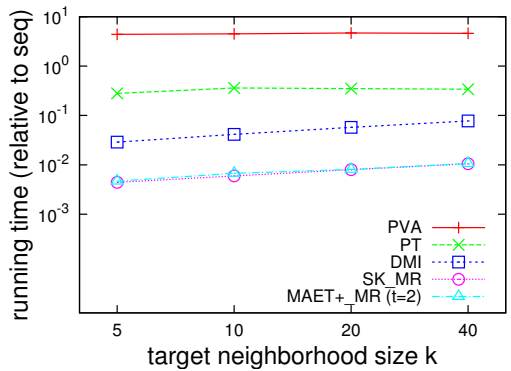

(d) FCT

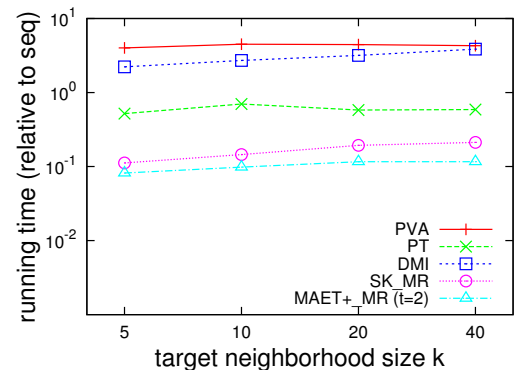

(e) ANN_SIFT

Figure 10: The results of varying $k$ on all tested datasets, with $\left|F^{\prime}\right|=8$. The results are exact, except for those of MAET+_MR. The average accuracies of MAET+_MR with $t=2$ are approximately $88 \%, 96 \%, 89 \%, 98 \%$ and $92 \%$ for ALOI, MNIST, Cortina, FCT and ANN_SIFT, respectively.

methods are quite stable with respect to $k$.

Finally, Figure 8 shows the preprocessing costs of all methods considered in our experimentation. While the preprocessing costs of our methods are substantial, the costs are justifiable in light of the improvements in performance at query time.

\section{Conclusion}

We have presented new solutions for the subspace similarity search problem based on multistep search, utilizing 1-dimensional lower-bounding distance functions associated with individual features for the efficient pruning of the search space. Among the possible choices of lower- 
bounding distance functions that can be used for the specified subspace, we select the one corresponding to the most discriminative query dimension. This is done by ranking the dimensions based on data variance. Two ranking strategies have been proposed: Single Ranking (SR) and Multiple Ranking (MR).

To apply the MR strategy, two parameter choices must be considered: the sample size $m$ and the variance neighborhood size $K$. Our experimental results indicate that the choice of $K$ does not greatly affect the performance, provided that $K$ is very small relative to the dataset size $|S|$. On the other hand, the sample size $m$ must be chosen with more care. As discussed in Section 3.2, the choice of $m$ can be guided by the analysis of sampling properties.

Our extensive experimental study showed that MR is able to perform better than SR for all datasets tested, and that our algorithms (SK_MR and MAET+_MR) are able to outperform their state-of-the-art competitors (PVA, PT and DMI) for a relatively wide range of subspace dimensions. Relative to SK_MR, we observed that for high subspace dimensionality, MAET+MR can achieve a significant improvement in running time at the expense of a slight drop in the accuracy of the query result.

One possible topic for future work on subspace similarity search is the investigation of alternatives to the variance in deciding the dimension within which lower-bounding distances are to be computed. In principle, any measure of the discriminability of single features could be substituted for the variance.

Another challenge for future research is the investigation of multidimensional lower-bounding distances for pruning, such as 2-dimensional or 3-dimensional distances. Although multidimensional distances would be expected to provide a tighter lower bound on the target distance, they cover fewer combinations of query dimensions, and thus are likely to be only of limited practicality. For example, if we consider using 2-dimensional lower-bounding distances for pruning, we must build an index for each combination of two dimensions in order to be able to handle all possible choices of target distances. Assuming $D$ dimensions in total, we would need to construct a quadratic number of indices - exactly $D(D-1) / 2$ - which is highly impractical when the value of $D$ is large. If instead we considered 3-dimensional or other higher-dimensional distances for pruning, the number of indices required would be prohibitively large. The utilization of multidimensional lower-bounding distances for pruning presents a considerable challenge for future research.

\section{Acknowledgments}

This work was partially supported by NSF under Grant 1241976 , and by JSPS under Kakenhi Kiban Research Grants (B) $15 \mathrm{H} 02753$ and (C) 24500135.

\section{References}

[1] K. S. Beyer, J. Goldstein, R. Ramakrishnan, U. Shaft, When is "nearest neighbor" meaningful?, in: ICDT '99: Proceedings of the 7th International Conference on Database Theory, Springer-Verlag, London, UK, 1999, pp. 217-235.

[2] V. Pestov, On the geometry of similarity search: dimensionality curse and concentration of measure, Inf. Process. Lett. 73 (1-2) (2000) 47-51. doi:http://dx.doi.org/10.1016/S0020-0190(99)00156-8.

[3] R. Weber, H.-J. Schek, S. Blott, A Quantitative Analysis and Performance Study for Similarity-Search Methods in High-Dimensional Spaces, in: Proc. Intern. Conf. on Very Large Data Bases, 1998, pp. 194-205.

[4] H.-P. Kriegel, P. Kröger, A. Zimek, Subspace Clustering, WIREs Data Mining and Knowl. Discov. 2 (4) (2012) $351-364$. 
[5] R. Kohavi, G. H. John, Wrappers for Feature Subset Selection, Artif. Intell. 97 (1-2) (1997) 273-324.

[6] M. Behrisch, F. Korkmaz, L. Shao, T. Schreck, Feedback-Driven Interactive Exploration of Large Multidimensional Data Supported by Visual Classifier, in: Proc. 2014 IEEE Conf. on Visual Analytics Science and Technology (VAST), 2010, pp. 43-52.

[7] C. G. Healey, B. M. Dennis, Interest Driven Navigation in Visualization, IEEE Trans. Vis. Comput. Graph. 18 (10) (2012) 1744-1756.

[8] H. Samet, Foundations of Multidimensional and Metric Data Structures, Morgan Kaufmann, 2006.

[9] F. Korn, N. Sidiropoulos, C. Faloutsos, E. Siegel, Z. Protopapas, Fast Nearest Neighbor Search in Medical Image Databases, in: Proc. Intern. Conf. on Very Large Data Bases, 1996, pp. 215-226.

[10] T. Seidl, H.-P. Kriegel, Optimal Multi-Step $k$-Nearest Neighbor Search, in: Proc. Intern. Conf. on Management of Data, 1998, pp. 154-165.

[11] M. Houle, X. Ma, M. Nett, V. Oria, Dimensional Testing for Multi-step Similarity Search, in: Proc. Intern. Conf. on Data Mining, 2012, pp. 299-308.

[12] M. E. Houle, X. Ma, V. Oria, J. Sun, Efficient algorithms for similarity search in axis-aligned subspaces, in: 7 th International Conference on Similarity Search and Applications (SISAP), 2014, pp. 1-12.

[13] H.-P. Kriegel, P. Kröger, M. Schubert, Z. Zhu, Efficient Query Processing in Arbitrary Subspaces Using Vector Approximations, in: Proc. Intern. Conf. on Scientific and Statistical Database Management, 2006, pp. $184-190$.

[14] T. Bernecker, T. Emrich, F. Graf, H.-P. Kriegel, P. Kröger, M. Renz, E. Schubert, A. Zimek, Subspace Similarity Search Using the Ideas of Ranking and Top- $k$ Retrieval, in: Proc. ICDE Workshop DBRank, 2010, pp. 4-9.

[15] T. Bernecker, T. Emrich, F. Graf, H.-P. Kriegel, P. Kröger, M. Renz, E. Schubert, A. Zimek, Subspace Similarity Search: Efficient $k$-NN Queries in Arbitrary Subspaces, in: Proc. Intern. Conf. on Scientific and Statistical Database Management, 2010, pp. 555-564.

[16] A. Guttman, R-trees: a Dynamic Index Structure for Spatial Searching, in: Proc. Intern. Conf. on Management of Data, 1984, pp. 47-57.

[17] X. Lian, L. Chen, Similarity Search in Arbitrary Subspaces Under $L_{p}$-Norm, in: Proc. Intern. Conf. on Data Engineering, 2008, pp. 317-326.

[18] M. Houle, H. Kashima, M. Nett, Generalized Expansion Dimension, in: Proc. ICDM Workshop PTDM, 2012, pp. 587-594.

[19] D. R. Karger, M. Ruhl, Finding Nearest Neighbors in Growth-Restricted Metrics, in: STOC, 2002, pp. 741-750.

[20] V. Chvátal, The Tail of the Hypergeometric Distribution, Discrete Mathematics 25 (1979) 285-287.

[21] J. M. Geusebroek, G. J. Burghouts, A. W. M. Smeulders, The Amsterdam Library of Object Images, International Journal of Computer Vision 61 (1) (2005) 103-112.

[22] N. Boujemaa, J. Fauqueur, M. Ferecatu, F. Fleuret, V. Gouet, B. L. Saux, H. Sahbi, IKONA: Interactive Generic and Specific Image Retrieval, in: Proc. Intern. Workshop on Multimedia Content-Based Indexing and Retrieval, 2001.

[23] Y. LeCun, L. Bottou, Y. Bengio, P. Haffner, Gradient-Based Learning Applied to Document Recognition, Proc. IEEE 86 (11) (1998) 2278-2324

[24] K. Rose, B. S. Manjunath, The Cortina Data Set, http://www.scl.ece.ucsb.edu/datasets/index.htm.

[25] A. Asuncion, D. J. Newman, UCI Machine Learning Repository, http://www.ics.uci.edu/ mlearn/MLRepository.html.

[26] H. Jégou, R. Tavenard, M. Douze, L. Amsaleg, Searching in One Billion Vectors: Re-rank with Source Coding, in: Proc. Intern. Conf. on Acoustics, Speech and Signal Processing, 2011, pp. 861-864.

[27] D. G. Lowe, Distinctive Image Features from Scale-Invariant Keypoints, International Journal of Computer Vision 60 (2) (2004) 91-110. 